Investigaciones Fenomenológicas, n. 9, 2012, 407-429.

e-ISSN: $1885-1088$

\title{
Phantasia y PERCepción en MARC RICHIR*
}

\author{
Alexander Schnell \\ Université Paris-Sorbonne (Paris IV), Francia \\ alex.schnell @gmail.com
}

Resumen: El presente estudio busca clarificar el papel de la phantasía en la refundación de la fenomenología transcendental de Marc Richir (en particular desde Phénoménologie en esquisses, de 2000). A partir del análisis de la phantasia y de la imaginación -que toma apoyo en las Lecciones de Husserl de 1904/1905- el autor pone de manifiesto la relación de la phantasía tanto con la percepción como con el lenguaje, al tiempo que trata de elucidar el estatuto temporal de dichos elementos.

Palabras clave: Apercepción, imaginación, lenguaje, percepción, Phantasía, temporalidad, transposición arquitectónica.

\begin{abstract}
The aim of this paper is to clarify the role of phantasía in Marc Richir's refoundation of transcendental phenomenology (especially since Phénoménologie en esquisses, 2000). Beginning with the analysis of phantasía and imagination -that lies upon Husserl's Lecture of 1904/05- the author highlights the relationship of phantasía both with perception and language, as he tries to understand their temporal status.
\end{abstract}

Keywords: Apperception, architectonical transposition, imagination, language, perception, Phantasía, temporality.

Con la publicación, en el año 2000, de Phénoménologie en esquisses. Nouvelles fondations", la "refundación" richiriana de la fenomenología no sólo halla su primera ejemplificación concreta, sino también su "asiento fundamental". Richir explora, efectivamente, el campo de la phantasía, que sustituirá, como

\footnotetext{
${ }^{1}$ Marc Richir, Phénoménologie en esquisses. Nouvelles fondations, Grenoble, J. Millon, 2000 (de ahora en adelante citado "PENF").

\begin{abstract}
* NdT: Este texto es una leve remodelación del capítulo segundo de la $1^{a}$ parte del libro de Schnell sobre Marc Richir titulado Le sens se faisant. Marc Richir et la refondation de la phénoménologie transcendentale, Ousía, Bruselas, 2011. Entre otras cosas, hemos obviado, en esta versión del texto, algunas referencias internas a otros capítulos del libro y, por el contrario, nos hemos esforzado por explicitar algunas menciones a desarrollos ya efectuados. En el número 34 de la revista Eikasia, una detallada tabla analítica de contenidos de este libro de Schnell de inminente publicación: http://www.revistadefilosofia.com/34-17.pdf y que, por lo demás, ofrece un buen panorama de algunos temas relativos a los desarrollos recientes de la fenomenología richiriana. También se puede consultar con provecho la bibliografía de y sobre Richir elaborada por Gérard Bordé: http://www.revistadefilosofia.com/34-14.pdf.
\end{abstract} www.revistadefilosofia.com, el primero de los dos números dedicado a Marc Richir, puede consultarse
} 
veremos, al de percepción como registro "fundante" (pero en el sentido de una "base" y no de un "fundamento") de esta fenomenología refundada. Así pues, preguntémonos, en primer lugar, ¿qué es la phantasía?

En la tercera parte del importante Curso impartido en $1904 / 05^{2}$, y titulado "Elementos principales de la fenomenología y de la teoría del conocimiento (Hauptstücke aus der Phänomenologie und Theorie der Erkenntnis)", Husserl había introducido la distinción entre dos tipos fundamentales de la conciencia imaginante, es decir, entre dos formas de "poner en imagen (verbildlichen)" el objeto al que dicha conciencia se remite. Si esta "imaginación" (en el sentido propio del término), o esta aprehensión que "pone en imagen (verbildlichend)", obra en el nivel de las representaciones de imagen "externas" (cuadros y fotografías), entonces se trata de la imaginación en sentido estricto; cuando, por el contrario, es relativa a las representaciones de imagen "internas", que carecen de dicho "soporte" exterior y donde ninguna "imagen", ninguna representación objetiva (sea cual sea) interviene, entonces nos las hemos con la "phantasía (Phantasie) ${ }^{\prime \prime 3}$. Lo que funda esta distinción reside en que, en el caso de la imaginación, existe una intencionalidad específica que mienta un Bildobjekt a través del cual se da el Bildsujet (como ocurre, por ejemplo, con el dibujo de una torre (=Bildobjekt) que "representa" la torre Eiffel (=Bildsujet)), mientras que, en el caso de la phantasia, dicho Bildobjekt falta, no se da. He aquí la alternativa que, para el caso de la phantasia, se desprende de este análisis: o bien es preciso, a pesar de todo, encontrarle a la phantasia una suerte de Bildobjekt (para no poner así en duda su carácter intencional ${ }^{4}$ ), o bien habrá que reconocer que la phantasia es de carácter no intencional. En las Lecciones de 1904/05, Husserl parece debatirse entre ambas posibilidades -y finalmente inclinarse por la primera (por no poder resolverse a poner en tela de juicio, dentro de su comprensión de la fenomenología, la prioridad absoluta acordada a la intencionalidad). M. Richir, por su parte, no sólo extrae todas las consecuencias de la segunda rama de la alternativa, sino que incluso hará de ella la matriz de toda relación al "objeto", y ello aguas arriba [en amont] y más acá de la

\footnotetext{
${ }^{2}$ Ver el volumen XXIII de Husserliana: Phantasie, Bildbewusstsein, Erinnerung (1898-1925), E. Marbach (ed.), La Haye, M. Nijhoff, 1980; Phantasía, conscience d'image, souvenir, tr. fr. por J.-F. Pestureau, Grenoble, J. Millon, 2002.

${ }^{3}$ Ver Alexander Schnell, Husserl et les fondements de la phénoménologie constructive, Grenoble, J. Millon, 2007, capítulo III.

${ }^{4}$ Lo que supone, claro está, que toda relación intencional mienta un objeto, es decir, en lenguaje richiriano, que es "dóxico".
} 
percepción objetivante. En efecto, M. Richir identifica a la phantasia como una "dimensión salvaje" ${ }^{5}$, más acá de la diferencia entre realidad e irrealidad - y, de haber intencionalidad, ésta procede, de entrada y originariamente, de la "transposición arquitectónica" de la phantasia en imaginación. En estas líneas se tratará de clarificar esta "transposición arquitectónica" que traduce el "nacimiento" de lo "dóxico" (y que caracteriza tanto a la imaginación como a la percepción) a partir de lo "no dóxico" (característico de la phantasia).

Uno $^{6}$ de los objetivos fundamentales de Phénoménologie en esquisses consiste, en primer lugar -tanto a través de una crítica de la metafísica como de una revisión de todas las desviaciones metafísicas en el seno mismo del campo de la "fenomenología"7 - en llevar a cabo una radicalización de la fenomenología, radicalización que ha de respetar la idea de que "hay que tomar la fenomenología no ya como una doctrina, sino como un conjunto de problemas y cuestiones cuya interrogación ha de proseguirse con arreglo a un método" ${ }^{\prime 8}$. Dicha radicalización exige pues una "refundición [refonte]" de la fenomenología; refundición cuyo aporte fundamental reside, efectivamente, en la refundación [refondation] de la estructura de la intencionalidad husserliana. Al retomar los análisis relativos al estatuto de los fenómenos como "nada sino fenómenos [rien que phénomènes]" (pertenecientes a la esfera fenomenológica más "arcaica") -que no son fenómenos de nada más que de sí mismos, más acá de, o, como dice M. Richir, "transcendiendo" lo real, lo fantástico, lo efectivo, etc. ${ }^{9}$, y que se encuentran en una relación de "parpadeo" con una u otra estructura intencional; estructura intencional que, los transpone, una y otra vez, arquitectónicamente $-M$. Richir ha sido conducido a lo que también denomina una "fenomenología nova methodo"10. Esta tentativa de erigir "nuevas fundaciones" es tarea que se le impone a M. Richir, precisamente en aras a establecer en

${ }^{5}$ PENF, p. 85.

${ }^{6}$ El presente texto es una versión reelaborada del segundo capítulo de la segunda parte de nuestra obra La genèse de l'apparaître, Études phénoménologiques sur le statut de l'intentionnalité, Beauvais, Mémoires des Annales de Phénoménologie, 2004 www.annalesdephenomenologie.org.

7 M. Richir se ha justificado en varias ocasiones sobre el particular. Cfr., por ejemplo, el Prefacio de Phénoménologie en esquisses, sobre todo las pp. 7-22; ver también "Métaphysique et phénoménologie: Prolégomènes pour une anthropologie phénoménologique", en E. Escoubas, B. Waldenfels (éds.), Phénoménologie Française et Phénoménologie Allemande. Deutsche und Französische Phänomenologie, Paris, L'Harmattan, 2000, pp. 116-121. Incluimos aquí el vínculo internet a este artículo "Métaphysique et phénoménologie. Prolégomènes pour une anthropologie phénoménologique".pdf disponible a través de la página sobre la fenomenología de Richir elaborada por Sacha Carlson: www.laphenomenologierichirienne.org

8 PENF, p. 478.
9 Ibidem, p. 480.
${ }^{10}$ Ibidem, p. 20. 
qué medida la Stiftung de phantasia (con la temporalización que le pertenece en propio) desempeña un papel decisivo en el seno de la esfera "primitiva" o "arcaica", y dando cuenta así del hecho de que la percepción objetivante -con su temporalidad correspondiente- no es sino la expresión de una estructura que erige en "simulacro ontológico"11 lo que, en realidad, no es sino del orden de una institución (simbólica) (Stiftung) particular.

El proyecto de M. Richir parte de las consecuencias de la enseñanza husserliana de que toda mención de objeto es una apercepción (Apperzeption) ${ }^{12}$, es decir, que el proyecto richiriano se toma enteramente en serio la idea de que hay un "exceso de la intención en la propia intención"13 o de que la Darstellung (figuración) intuitiva de aquello de lo que la apercepción es apercepción jamás puede "saturar" la intuición"14. (Expresión célebre de este hecho es que, por ejemplo, en la percepción de un objeto transcendente, este último sólo se da bajo la forma de "escorzos" o de "adumbraciones [adombrations]" (Abschattungen), apareciendo, sin embargo, como objeto y no como puro escorzo). Ahora bien, si así ocurre, hemos de emplearnos en buscar el origen de las apercepciones $^{15}$, es decir, emplearnos en determinar el origen de ese "vacío intuitivo" sin el cual no habría intencionalidad. La hipótesis de M. Richir radica entonces en que "este vacío es la 'memoria' o la 'huella' de una transposición arquitectónica operante en el nivel mismo de la intuición ${ }^{16 " . ~ E s t a ~ " t r a n s p o s i c i o ́ n ~ a r-~}$ quitectónica" implica tres cosas:

1) Exige, según M. Richir, volver a descender a las profundidades, más acá del sentido instituido (gestiftet) -sedimentado con la habitualidad- y también más acá de la apercepción como "sentido intencional", para así desvelar, como

${ }^{11}$ Marc Richir denomina "simulacro ontológico" a la estructura que erige tal o cual fenómeno en matriz transcendental de toda aparición y de todo fenómeno; matriz que otorga ser a una "vida" que no lo tiene. Ibidem, p. 480.

${ }^{12}$ Cfr., por ejemplo, Husserliana XI, p. 10. En esa misma Introduction a las Lecciones sobre el análisis de las síntesis pasivas, Husserl introduce asimismo una acepción un poco diferente de la apercepción, a saber, la de la apercepción transcendental, y que designa la operación de conciencia que atribuye a los datos hiléticos inmanentes la función de figurar (darstellen) entidades "transcendentes" y objetivas (ibidem, p. 17).

${ }^{13}$ Husserl habla a este propósito de un "Über-sich-hinaus-meinen" (de un "exceso" o, según la traducción de Peiffer y Levinas, de un "dépassement") que reside en toda intención de conciencia. Cfr. la II ${ }^{a}$ Meditación cartesiana, Vrin, 1947, 1992, § 20, p. 86 (Husserliana I, p. 84).

${ }_{14}$ PENF, p. 39ss, p. 44.

${ }^{15}$ Ibidem, p. 40. Constatamos así la proximidad entre el proyecto de M. Richir y la fenomenología genética husserliana tal y como se la presenta en "Statische und genetische phänomenologische Methode", Husserliana XI, pp. 336-345. Cfr. a este propósito el capítulo IV, sección VIII, de La genèse de l'apparaître, op. cit.

${ }^{16}$ PENF, p. 44. 
hemos subrayado, la formación (Bildung) del "sentido haciéndose 17 ": en esta transposición "que sólo cabe poner de relieve arquitectónicamente ${ }^{18 "}$, está pues en juego, en sentido inversa, el paso del registro de la Bildung del sentido al de su Stiftung, lo cual abre la vía a una exploración de la "génesis de las apercepciones" (génesis en el sentido de la Krisis de Husserl).

2) Esta transposición arquitectónica, origen del vacío intuitivo recién mentado, muestra, por otro lado, que la estructura uniforme de la temporalización en flujo del presente vivo provisto de sus retenciones y de sus protenciones no es una estructura universal (en efecto, tan sólo corresponde "a un cierto tipo de transposición arquitectónica, al término de la cual se instituye la apercepción estable de un objeto duradero"199); por consiguiente, todas las estructuras "intencionales" deben, cada vez, ser consideradas junto a las estructuras de temporalización/espacialización que les son específicas. La cuestión de la naturaleza de la temporalización (y de la espacialización) de cada tipo de institución ( $y$, muy en especial, la puesta en tela de juicio del estatuto "matricial" de la temporalización de la institución perceptiva) es pues un problema absolutamente crucial para M. Richir.

3) Advertimos entonces que no es ya cuestión, aquí, de la constitución de una objetividad, sino, una vez más, de la formación $-\mathrm{y}$, después, de la institución- del sentido (lo que viene a ser, para M. Richir, un punto de vista más fiel a la enjundia [teneur] fenomenológica de la intencionalidad -la cuestión de la constitución siempre está, en definitiva, fundada en la de la institución y, a fortiori, en la de la formación del sentido). Procediendo de este modo a una "ampliación de la fenomenología"20, M. Richir substituye el problema de la constitución de la objetividad por el de la consideración del lenguaje fenomenológico (de los fenómenos de lenguaje y de los Wesen [seres] de lenguaje), testimonio de la formación del sentido haciéndose y de su enunciación (o de su expresión) en una "lengua". La siguiente cita expresa y resume perfectamente estos tres puntos que acabamos de poner en claro:

[...] la intencionalidad que obra en toda apercepción ha de tomarse, en tanto que mención de sentido intencional, como la huella del modo en que se efectuó, a ciegas, la

\footnotetext{
${ }^{17}$ Ibidem, p. 21.

${ }^{18}$ Ibidem, p. 43.

${ }^{19}$ Ibidem, p. 45.

${ }^{20}$ Ibidem, p. 43.
} 
transposición arquitectónica del lenguaje a la lengua en la Stiftung [...] de la apercepción $n^{21}$.

El hecho de que esta huella (detectada gracias al modo propio de temporalización/espacialización de la intencionalidad; intencionalidad instituida, a su vez, en la Stiftung de la apercepción) sea diferente cada vez y según las apercepciones sean de percepción, de imaginación, de phantasia, de recuerdo, etc., establece el marco o el programa de lo que M. Richir se propone desarrollar en Phénoménologie en esquisses.

El "primado de la percepción", característico de la fenomenología husserlina, no ha permanecido inadvertido para numerosos comentadores de Husser ${ }^{22}$. Con todo, todavía hace falta comprender el vínculo que existe entre la manera en que se instituye la apercepción de percepción y la estructura de la intencionalidad husserliana. Veremos que esta relación es una relación temporal. Seguiremos a partir de ahora punto por punto el análisis que hace M. Richir de la apercepción perceptiva, lo que nos permitirá apreciar, más adelante, qué tipo de temporalidad se exhibe en esta última.

La apercepción perceptiva está caracterizada por una "contradicción ${ }^{23 "}$ que se expresa mediante el hecho de que aunque la relación intencional jamás se halla saturada, el objeto que se da en ella se da, con todo, "en persona"; dicho de otro modo, a pesar de que el objeto nunca aparezca sino bajo la forma de "escorzo", el objeto es percibido como siendo el objeto mismo. ¿Cuál es entonces el fundamento temporal de esta apercepción "por escorzos"? O, enunciado en otros términos, ¿cómo "se abre" este desarrollo perceptivo (del objeto) como flujo temporal continuo? Si la apercepción es la aparición de un objeto que sin embargo no aparece sino unilateralmente; si, por consiguiente, la aparición de un objeto implica co-aparición, o co-consciencia de otras apariciones no intuicionables en el presente, entonces esas otras apariciones, que no son actualmente visibles, están prescritas con arreglo a horizontes llamados a "ser plenificados" por apariciones que, por consiguiente, habrán de ser efectivamente intuicionables. Lo decisivo aquí radica en que estos horizontes afectan direc-

${ }^{21}$ Ibidem, pp. 47ss.

22 Esto es evidentemente cierto, en primer término, de la Phénoménologie de la perception de Merleau Merleau-Ponty, pero también de la obra de Gerard Granel que, al menos sobre este asunto, marcó una época: Le Sens du temps et de la perception chez E. Husserl, Paris, Gallimard, 1968; ver también Klaus Held, Lebendige Gegenwart, La Haye, M. Nijhoff, 1966, p. 8.

${ }^{23} \mathrm{Cfr}$. Husserliana XI, p. 3. 
tamente al sentido de ser-así del objeto percibido; sentido que, de este modo, puede cambiar en función de cambios en aquéllos. ¿Cómo hemos de entender esto? Lo cierto es que dichos horizontes tienen, justamente, una función fundamentalmente temporalizante. Prefiguran nuevas apariciones posibles: esta prefiguración (Vorzeichnung) implica a la vez una herencia pasada (la "memoria" del objeto operando como pasado del objeto que continua siendo mentado en vacío, incluso cuando las apariciones ya han caído fuera del campo de las retenciones actuales) y una anticipación futura (el propio término "prefiguración" lo dice de forma explícita) ${ }^{24}$. M. Richir puede entonces determinar el movimiento de la figurabilidad (Darstellbarkeit) intuitiva al hilo de su figuración (Darstellung) en la apercepción perceptiva:

[...] el a parte post [l'après coup] y el a parte ante [l'avant coup] se reajustan permanentemente en el seno mismo del sentido de ser-así del objeto, es decir, en el seno del sentido intencional sostenido por la apercepción instituida del mismo objeto: es lo que hace [que] esta apercepción como apercepción de una mismidad no sea sino este reajuste mismo, este acuerdo en movimiento de un pasado y de un futuro vacíos de apariciones o de intuiciones. ${ }^{25}$

Comprendemos ahora el vínculo que existe entre el hecho de que la aparición actual sea una "adumbración" y la institución del decurso temporal: la aparición presente "remite, por su sentido intencional de aparición del objeto como de un mismo objeto, hacia el pasado y el futuro, hacia un vacío de aparición o de intuición, y ello por cuanto su aparente plenitud intuitiva en el Jetzt del decurso [écoulement] continuo instituido del tiempo está envuelto y penetrado de no-intuitividad $[\ldots]^{\prime 26}$. Dicho de otro modo, la percepción actual y concreta del objeto por un lado, y, por el otro, el "vacío intuitivo", origen de lo que se expresa en el hecho de que la aparición del objeto se haga "por escorzos", están en una relación de mediación retencional y protencional que reclama "plenificar" (o "decepcionar") este vacío - proceso en que se basa lo que constituye el "transcurso [cours] de la experiencia".

\footnotetext{
${ }^{24}$ Constatamos aquí un entreveramiento [enchevêtrement] entre las protenciones y las retenciones analizado por Husserl en el texto $n^{\circ} 2$ de Husserliana XXXIII; para mayores detalles, cfr. nuestra obra Temps et phénomène. La phénoménologie husserlienne du temps (1893-1918), Hildesheim, Olms, 2004, pp. 136ss. Ver también PENF, pp. 190ss.

${ }^{25}$ PENF, pp. 52ss.

${ }^{26}$ Ibidem, p. 53.
} 
Baste esta descripción para caracterizar, en primera aproximación, la apercepción perceptiva. Ahora bien, la institución de esta apercepción perceptiva es la de un objeto que persiste, en su presencia (Vorhandensein), a través de su decurso temporal. Es la expresión de un cierto tipo de transposición; transposición al cabo de la cual se instituye una apercepción estable de un objeto duradero (por lo tanto, la apercepción perceptiva del Vorhandensein [estarpresente] heideggeriano). M. Richir evidencia dicha apercepción como lo que, según Husserl, está a la base del "fenómeno originario" (Urphänomen) de todo fenómeno. Tras haber analizado la naturaleza y el estatuto de la apercepción perceptiva tal como M. Richir la detecta, explícitamente, en el texto de Husserl, podemos, ahora, reconstituir su propia interpretación de esta última (una interpretación que cumple hacer dado el carácter las más veces implícito de los análisis husserlianos sobre este tema) ${ }^{27}$. Aislemos entonces los tres puntos esenciales que caracterizan de forma exacta la institución de la apercepción perceptiva (así como la temporalidad que le corresponde) según esta interpretación, y de la que M. Richir se ocupa; interpretación contenida en el $\S 2$ de la Introducción a su obra Phénoménologie en esquisses:

1) En la apercepción perceptiva se instituye, como se ha dado a entender, la permanencia o también el "estar-presente (Vorhandensein)" del objeto percibido.

2) Además, la apercepción perceptiva se caracteriza por un reajuste "simultáneo" entre -una y otra vez- el surgimiento de un presente sin cesar nuevo y, al mismo tiempo, la retención (siempre modificada) del presente recién pasado. La relación entre este reajuste y el Vorhandensein es de mediación circular entre ambos momentos.

3) Se instituye por fin, en la apercepción perceptiva, la continuidad del tiempo o, más exactamente, del "presente vivo" provisto de sus protenciones y retenciones.

1) Antes de nada -y esto es capital- el tiempo inmanente es, para M. Richir, del orden de una institución "instantánea" que, a partir de un presente

\footnotetext{
${ }^{27}$ Marc Richir afirma, en efecto, que "Husserl jamás fue demasiado explícito sobre el particular" y que eso exige "interpretar" lo que ello implica para el estatuto de la temporalidad de la institución perceptiva. PENF, p. 53.
} 
dado, instituye el tiempo como decurso de ese mismo presente y, a través de ello, la percepción del objeto (dado que dicha percepción sólo se hará posible en virtud del horizonte interno, y que es un horizonte protencionalretencional $)^{28}$. La percepción del objeto se ve, de ese modo, mediatizada por una institución que es fundamental y exclusivamente temporal (la serie temporal hace posible la serie de las percepciones). Lo que obra de intermediario entre el decurso del presente (y su modificación) y la percepción del objeto, es la cosa "en la percepción", constituida "en carne y hueso (in seiner Leibhaftigkeit)" y en su permanencia a través del decurso del tiempo.

2) Pero, ¿cómo se constituye esa permanencia misma? Sucede que esta "institución instantánea" -actual y concreta- es "al mismo tiempo"29 (expresión en realidad ilegítima puesto que usa términos de la temporalidad constituida cuando, en rigor, nos encontramos, aquí, en la esfera de la temporalidad constituyente) institución de un pasado y de un futuro perceptivos ajustados al surgimiento sin cesar renovado de un presente actual. Y la clave de la apercepción perceptiva debe entonces buscarse en el hecho de que, del mismo modo, y no ya en el plano de la serie temporal, sino, esta vez, en el de la percepción, el continuo de las apariciones presentes se ajusta al de las apariciones que fluyen [s'écoulant] de manera retencional hacia el pasado (sucediendo otro tanto con las apariciones protencionales). Hagamos notar que el reajuste "simultáneo" entre el surgimiento de un presente nuevo y la retención de un presente precedente está en una relación de mediación circular $^{30}$ con la "permanencia" del objeto (que está en el origen de su "estar-presente (Vorhandensein)"): por un lado, la percepción como apercepción reajusta permanentemente el pasado y el futuro perceptivos ya que la institución instantánea de la serie temporal es también institución de la serie de las percepciones (o, en otros términos, porque la institución temporal es ipso facto institución perceptiva); por otro lado, el sentido de ser del Vorhandensein procede, precisamente, de este reajuste permanente.

\footnotetext{
${ }^{28}$ Marc Richir resume esta idea como sigue: "Podemos interpretar esto alegando que la Stiftung, como institución instantánea (simbólica), en el acto de un presente que, a partir de entonces, instituye el tiempo como decurso del mismo presente es, a la vez, institución de la misma percepción del mismo objeto, luego institución como mismo del sentido de ser y de ser-así de dicho objeto". PENF, p. 53.

${ }_{29}$ Se trata del "al mismo tiempo" de un fenómeno de lenguaje allanado [aplati], nivelado.

30 Marc Richir pone explícitamente de manifiesto esta circularidad en el Capítulo I de la IIa sección de Phénoménologie en esquisses, p. 185.
} 
3) Comprendemos entonces cómo se instituye, de ese modo, un decurso temporal continuo. Si la institución de la serie temporal ( $y$, después, de la apercepción perceptiva) es un continuum, si este último se ajusta a un continuum de apariciones surgiendo y modificándose de continuo en retenciones, la entera apercepción perceptiva no conoce "falla" discontinua alguna. He ahí el vínculo que, al fin y al cabo, existe entre esta institución de la apercepción y la de la continuidad temporal: "Lo propio de esta Stiftung [...] es abrir el desarrollo [déroulement] perceptivo como flujo temporal [...] continuo de las apariciones del mismo objeto" ${ }^{31}$; esto significa que la Stiftung aperceptiva no sólo lo es del "vacío" aperceptivo (que remitía a las protenciones y a las retenciones) sino que también lo es, como hemos comprobado, de la propia continuidad.

Para poder captar el sentido de la apercepción de phantasia, es necesario, en primer lugar, reconstruir lo esencial de la crítica richiriana de la concepción husserliana de la institución de la apercepción perceptiva.

Si bien es cierto que, en los Manuscritos de Bernau, Husserl responde a ciertos problemas muy urgentes (por ejemplo, al del estatuto de la impresión originaria, al del papel de la protencionalidad, al del estatuto del "flujo absoluto de la conciencia", etc.), problemas que se le habían planteado a su fenomenología del tiempo tal y como la conocemos desde la publicación, en 1928, de las Lecciones sobre la conciencia interna del tiempo, esto no impide que no consiga restañar todas las heridas que ya desde el texto originario ( $y$ profundamente original) de las Lecciones de 1904/1905 permanecían abiertas. Los tres problemas esenciales e irreductibles aún remanentes se refieren, según M. Richir, a

1) la continuidad irreductible tanto de la temporalidad inmanente como de la temporalidad pre-inmanente ${ }^{32}$;

${ }^{31}$ PENF, p. 53.

32 ¿Por qué Husserl no prosiguió de una manera suficientemente consecuente el procedimiento de una doble reducción fenomenológica (Cfr. los Grundprobleme der Phänomenologie de 1910/11) permitiendo abandonar la "vía cartesiana" de la reducción (efectivamente, ya no hay rastro de la misma en las Meditaciones cartesianas)? Se debe, según László Tengelyi, a la concepción husserliana del "tiempo vivido" y, más en particular, a la "modificación continua", precisamente del presente al pasado (una tesis que Ricœur resume con esta pregnante fórmula: "la modificación precede a la diferencia", Temps et récit, op. cit., tomo III, p. 51), modificación que imposibilita una "formación de sentido" (Sinnbildung) que sobrepase la ipseidad del Ego hacia el Alter Ego y que así invalida, por ende, la posibilidad de "ampliar" la reducción fenomenológica (en el sentido de la "vía cartesiana") hacia lo que permite acceder a la "extrañeza (Fremdheit)" (Der Zwitterbegriff Lebensgeschichte, München / Paderborn, W. Fink, 1998, pp. 70-74; ver también, del mismo autor, las dos primeras secciones de la segunda parte de L'histoire d'une vie et sa région sauvage, Grenoble, J. Millon, 2005). 
2) el problema del reajuste entre el decurso retencional y el resurgimiento de cada nuevo presente;

3) el problema del estatuto formal de los análisis de la constitución de la temporalidad fenomenológica.

Una lectura del texto no 1 de Phantasia, conciencia de imagen, recuerdo ${ }^{33}$ permite mostrar que la temporalidad de phantasia pone definitivamente en duda el carácter exclusivo y universal de la continuidad del tiempo. La temporalidad de la phantasia es el ejemplo evidente que choca con la tesis según la cual cualquier tipo de temporalidad obedecería a la ley de la continuidad universal del tiempo. Como, por su parte, Fink $^{34}$ ya ha hecho notar este mismo aspecto, nos concentraremos, en lo que sigue, sobre los otros dos problemas, y lo haremos apoyándonos en los análisis de Phénoménologie en esquisses ${ }^{35}$. La exposición de estos problemas nos permitirá, a continuación, analizar las contribuciones de M. Richir a la fenomenología del tiempo.

El "enigma" de la institución perceptiva consiste en el acuerdo entre dos ritmos -el del surgimiento de un presente actual y el de una retención simultánea del presente precedente. Pero, ¿de veras están acordados de la manera tan estricta e infalible en que Husserl ${ }^{36}$ afirma que lo están? En realidad hay, "en un momento dado", ruptura entre ambos flujos, es decir que, con el surgimiento de un nuevo ahora, la "cola de cometa" de las retenciones ya no puede verse colmada por nuevas retenciones vivas (un estado de cosas que no significa sino, como M. Richir lo hace notar, el "quedar sumidas en el olvido"37).

¿Qué implica, entonces, este no acuerdo (al menos parcial) o este fracaso del reajuste entre ambos ritmos? Resulta que según esta forma de decurso, que es una forma intemporal (que llamamos "flujo absoluto" o "proceso originario") ningún contenido intrínseco a la propia temporalización tiene tiempo de entrar, viéndose, al punto, eclipsado por la repleción [comblement] del desajuste [écart] retencional (que se abre sin tregua) por el presente; presente

\footnotetext{
33 Edmund Husserl, Phantasía, conciencia de imagen, recuerdo, op. cit.

${ }^{34} \mathrm{Cfr}$. el último capítulo de nuestra obra En deçà du sujet. Du temps dans la philosophie transcendantale allemande, Paris, P.U.F., 2010

${ }^{35} \mathrm{Cfr}$. en particular el capítulo I de la IIa sección, pp. 185-203.

${ }^{36}$ Husserl no se desvía en ningún momento (tanto en los textos de Husserliana $X$ como en los de Husserliana XXXIII) de su "postulado" de la continuidad ( $y$, por ende, del reajuste infalible entre los dos ritmos de que aquí tratamos) de la forma de la temporalidad fenomenológica.

37 PENF, p. 201.
} 
que cae, a su vez y de inmediato, en retención. Dicho de otro modo, nada tiene tiempo de madurar, quedando así "a expensas [en souffrance]" o en el "sueño [sommeil]" de la potencialidad, ya que algo actual [de l'actuel] ocupa inmediatamente su lugar para, ello sólo, sumirse, a su vez, en la inactualidad, en la potencialidad del hábito y de la sedimentación ${ }^{38}$.

A pesar de que, contrariamente a lo que afirma M. Richir, sí que haya, en la citada estructura, "potencia (Potenz, dynamis) anterior e independiente del acto", no deja de ser cierto que "en él no se forma tiempo" y que éste no hace otra cosa que repetirse continuamente en su célula (presente provisto de sus retenciones y de sus protenciones)". Esto significa, como subraya M. Richir, que "el contenido del tiempo sólo puede proceder del afuera del propio tiempo, sólo puede ser recibido en la Urimpression que también es Urempfindung, y sólo puede ser aquello al albur de lo cual la célula se distribuye según sus intencionalidades transversal y longitudinal"39. Ahora bien, una vez notadas estas insuficiencias, podemos extraer las consecuencias en punto a esta ausencia de contenidos intrínsecos situados en el nivel de las intenciones: estas consecuencias, que parten de una teoría de la formación del sentido haciéndose permitirán entonces comprender cómo se instituye la apercepción perceptiva.

¿Qué significa exactamente la idea de que el contenido del tiempo no puede proceder sino de un "afuera" (aparente) del tiempo? Lo que parecía discurrir en el sentido de una crítica del carácter "formal" de los análisis husserlianos del tiempo se revela ahora como la rigurosa exigencia de una "superación", por así decirlo, de la esfera de la pura inmanencia. Sin embargo, contrariamente a los proyectos de Husserl y de Fink, M. Richir no toma la vía de un análisis constitutivo, sino que se ve obligado, siguiendo, por lo demás, a Desanti ${ }^{40}$, a dar cuenta no ya de la objetivación, sino de la enunciación en lenguaje de la apercepción perceptiva. Este "paso" -que, por lo tanto, ni es un análisis constitutivo, ni a fortiori, una deducción- se expresa, en M. Richir, en los términos de una "transposición arquitectónica" que tiene como "destino" la apercepción perceptiva y como "origen" la temporalidad del "sentido haciéndose". Conviene entonces precisar las dos "esferas" de esta transposición.

\footnotetext{
${ }^{38}$ Ibidem, p. 192. El subrayado es nuestro.

${ }^{39}$ Idem, p. 192.

${ }^{40}$ Ver el primer capítulo de la segunda parte de nuestra obra La genèse de l'apparaître, op. cit.
} 
Ya hemos observado que la temporalidad inmanente (la de la apercepción perceptiva) está desprovista de contenidos intrínsecos y que estos contenidos deben llegar desde un "afuera" (o al menos desde lo que aparece como dicho afuera). Esto entraña dos importantes consecuencias:

1) La forma del decurso continuo de la apercepción perceptiva, cuya permanencia y estabilidad objetuales están aseguradas en virtud de su propia for$m a$, requiere un contenido que no es otro que la "institución originaria" en que consiste la impresión originaria (o la sensación originaria).

2) En la percepción no hay, "propiamente hablando, lenguaje, es decir, elaboración temporalizante del sentido haciéndose": "el sentido se halla siempre en ella simbólicamente 'condensado' en clave de sentido intencional" y "en virtud de esta 'condensación' simbólica, que corto-circuita toda elaboración de lenguaje y que hace que su Historia no sea sino la de su sentido intencional instituido, la percepción acalla [rend muet], eclipsa en todo momento y mediante su contenido intuitivo cualquier esbozo [amorce] de sentido de lenguaje que pudiera insinuarse en su seno" ${ }^{\prime 41}$. Ahora bien, precisamente de esta elaboración de lenguaje del objeto de la percepción, habrá de dar razón una fenomenología del tiempo. La pregunta que entonces se plantea es la de cómo una transposición arquitectónica del sentido haciéndose es posible y cuál es la puesta en forma de los contenidos que ésta entraña -contenidos "que son (relativamente) extrínsecos a esa misma temporalización, que son recibidos 'desde fuera' ${ }^{\prime 42}$.

Situémonos, anticipando, sobre el propio plano de la formación de sentido (Sinnbildung), es decir, sobre el plano a partir del cual se opera, según M. Richir, la transposición arquitectónica. Si bien es imposible situar un presente propiamente dicho ( $y$ a fortiori un ahora puntual (Jetztpunkt)), cabe, sin embargo, cernirlo a parte post, al cabo de una "elaboración" protencional y retencional. Ahora bien, es importante no confundir estas retenciones y protenciones -que son sin presente asignable- con aquéllas, propias de la esfera inmanente, que M. Richir caracteriza (quizá no exactamente en conformidad con Husserl) como "actos". Más bien se trata de "entre-apercepciones" (de lenguaje) "en la medida en que se entre-aperciben en ellas trizas [lambeaux] del sentido

41 PENF, p. 193.
42 Idem.

Investigaciones Fenomenológicas, n. 9, 2012. 
haciéndose, Wesen de lenguaje, y donde no se aperciben, en cambio, objetos estables que fueran a su vez presentes ${ }^{\prime 43}$. Recordemos que la intención en la fase en curso de temporalizarse en presencia es pro-yecto o mención de sentido aún por hacer "que, en primer lugar, es sentido de lenguaje (fenomenológi-

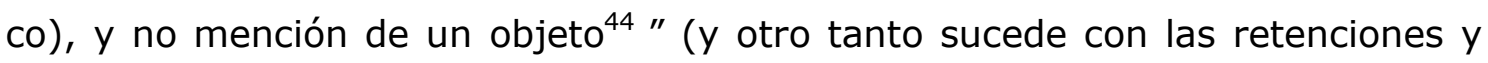
las protenciones).

Nos encontramos ahora en posición de comprender toda la amplitud de la institución de la apercepción de phantasia para la estructura de la intencionalidad husserliana.

Como lo habíamos señalado más arriba, la "representación" de phantasia procede de una Stiftung distinta a la de la representación de percepción. Es el resultado al que llega el propio Husserl al término del texto no 1 de Husserliana XXIII en que opone de una manera muy elocuente la percepción a la phantasia:

La percepción toma el apareciente como algo que es la cosa misma, es decir, como, justamente, algo que ella no modifica, que no imagina, que toma como siendo la cosa misma. La misma aparición puede ser la base de una conciencia de presentificación; se trata de la modificación. Sin embargo, esto no ha de comprenderse como si el apareciente estuviese dado, en primer lugar, de un modo no-modificado y como si la modificación sólo surgiese a parte post, reinterpretando, a la manera de una imagen, lo que está dado en presente como algo que no está dado. [...] Es algo que queda excluido a partir de nuestros análisis de la phantasia. ${ }^{45}$

Ahora bien, ¿qué es lo que caracteriza en propio a la phantasia respecto de la percepción, y cuál es su temporalización específica? Será la comparación entre sus contenidos de aprehensión respectivos (las "sensaciones" para la percepción y los "phantasmata" para la phantasia) lo que nos dé la respuesta a la pregunta:

El phantasma es una vivencia, pero no [como es el caso de la sensación] una vivencia que fuera tomada, de entrada, como presente, como siendo ello mismo, para, después, ser tomada por otra cosa. Si tomamos el phantasma por algo presente, es sólo por ser una parte integrante de la representación de phantasia, que sí es, por su parte, un presente. ${ }^{46}$

El punto esencial concierne por tanto al estatuto del "presente" del phantasma: éste ha de ser concebido como originariamente no presente. De hecho,

\footnotetext{
${ }^{43}$ Cfr., Marc Richir, L'expérience du penser, IV ${ }^{a}$ sección, pp. 445-448.

44 PENF, p. 194.

${ }^{45}$ Husserliana XXIII, p. 106.

${ }^{46}$ Idem.
} 
sólo está presente en la medida en que, a parte post, es transpuesto por la imaginación en parte sensible de la imagen que, por su parte, es un fictum, es decir simple nonada [un rien], fijada apenas un instante para verse, al punto, temporalizada en un presente con sus retenciones (en virtud de una "transposición arquitectónica"). ¿Qué quiere decir que la phantasia es "originariamente no presente"? Significa, y es algo esencial, que está desprovista de "representante" sensible asignable ${ }^{47}$.

¿Cuál es entonces la temporalidad específica de la apercepción de phantasia? La terminología fenomenológica, como Husserl lo subraya, por ejemplo, en el texto no 54 de Husserliana $X$, es tributaria de la objetividad constituida a pesar de esforzarse por dar cuenta de los fenómenos constituyentes. Ahora bien, puesto que la institución de phantasia se opone a la institución de percepción, constitutiva de la objetivación perceptiva, no nos extraña un ápice que, al menos en el orden del discurso, la caracterización de la apercepción de phantasia se haga primero negativamente (oponiéndola, precisamente, a la apercepción perceptiva). El campo de phantasia está en conflicto ("total") con el campo perceptivo: por consiguiente, "en la medida en que la percepción 'armónica', sin conflicto, constituye la aparición del presente actual, aquello con lo cual ésta entra en conflicto no está presente, es un no presente ${ }^{\prime 48}$. Será pues en virtud de dicho conflicto (M. Richir, citando a Husserl, habla, a este propósito, de una "tensión ${ }^{49 ")}$ como Husserl podrá decir que "lo apareciente [I'apparaissant] en la phantasia no está presente ${ }^{50}$ ". Tratemos ahora de caracterizar de forma positiva la institución de este apareciente de la phantasia.

M. Richir nos dispensa esta caracterización comentando el "texto crucial" de la página 78 de Husserliana XXIII ${ }^{51}$ : la diferencia fundamental entre la percepción y la phantasia reside en que la percepción constituye "al menos 'un momento'" de las impresiones o de las sensaciones presentes mientras que, en el caso de la phantasia, este "momento" no tiene lugar, está "corto-circuitado". Mientras que, en la percepción, el contenido de aprehensión (la sensación) está constituido como presente, no sucede otro tanto con el phantasma. "Ello implica [...] que la aparición de phantasia no es en sí misma presente, no tiene 'rea-

\footnotetext{
${ }^{47}$ A este propósito, Cfr., por ejemplo, Husserliana XXIII, Anejo XIII, p. 169.

${ }^{48}$ PENF, p. 77.

${ }^{49}$ Idem.

${ }^{50}$ Husserliana XXIII, p. 68.

${ }^{51}$ Cfr. PENF, p. 81.
} 
lidad de presente' $[\ldots]^{\prime \prime 2}$. Nos las habemos aquí con un régimen de temporalización distinto, lo cual Husserl confirma explícitamente en el $\S 38$ del texto $n^{\circ} 1$ de Husserliana XXIII:

En el caso de la phantasia no tenemos "presente" y, en ese sentido, tampoco objetoimagen. En la phantasia clara vivimos phantasmata y aprehensiones objetivantes que no constituyen [algo] que se sostuviese ahí, en presente, y que hubiese de funcionar, de entrada, como portador de una conciencia de imagen. La propia aparición carece por completo de cualquier relación al presente. ${ }^{53}$

Ahondemos en el análisis de la temporalización de la aparición de phantasia. Constatemos, para empezar, que M. Richir, criticando, en Husserl, el paradigma de la continuidad del tiempo, en lugar de oponerle, simplemente, una discontinuidad abstracta, aún tributaria de aquello a lo que se opone, se toma en serio la dimensión continua del tiempo (su "fluidez"), corrigiendo así, en cierto modo desde el interior de la concepción husserliana, las insuficiencias de esta última. ¿Qué es lo que caracteriza, en efecto, el decurso continuo del presente vivo provisto de sus retenciones y de sus protenciones? Es el hecho de que en esta continuidad se opera, con el surgimiento de cada nuevo ahora, una parada [arrêt], un suspenso de este decurso que constituye, en su fijeza, un presente; presente al punto modificado y retenido. Ahora bien, una descripción fenomenológica fiel de la institución del decurso temporal -y testimonio de ello es, precisamente, la temporalidad de la phantasia- permitiría advertir que, en la "fluidez" de la temporalidad originaria, no hay fijación de un presente, sino más bien una "temporalización en presencia sin presente asignable" (y que es todo menos pura y simple ausencia), y que, por lo tanto, hay una temporalización en la que las apariciones de phantasia surgen y se desvanecen originariamente en retenciones $y$ en protenciones, pero en retenciones y protenciones que no lo son de un presente vivo, que se distribuyen originariamente como tales en la propia presencia, es decir, que caen dentro de la presencia, presencia que ha de tomarse, por ende, como fase -fase no ya de un presente en flujo como en Husserl ${ }^{54}$, sino fase en la que se forma, sin presente asignable [...],

\footnotetext{
52 Ibid., p. 81 (el subrayado es de M. Richir). La inscripción de la aparición de phantasia en la "relación temporal" ("objetiva") sólo puede hacerse "a parte post", en la rememoración o, como sostendrá Husserl, merced a la reflexión (Cfr. Husserliana XXIII, p. 79).

53 Husserliana XXIII, p. 79, citado por M. Richir, PENF, p. 82.

${ }^{54} \mathrm{La}$ acepción husserliana de la noción de "fase" está desarrollada en el texto no 47 de Husserliana $\mathrm{X}, \mathrm{pp}$. 317ss. Así y todo, hagamos notar que hay un pasaje en el texto $\mathrm{n}^{\circ} 5$ de Husserliana XXXIII en que Husserl accede a una noción de fase que integra una dimensión fluyente: "Lo que se dice de las
} 
tiempo como tiempo de la presencia y del al-mismo-tiempo. Es pues necesario, para adentrarse en las paradojas de la phantasia, concebir una presencia que no sea presencia de uno o varios presentes ${ }^{55}$.

De este modo accedemos al verdadero significado de la presentificación, y que no consiste en volver algo presente (acaso sólo en un sentido derivado), sino en temporalizar en presencia lo que, originariamente, no está presente. En los términos de M. Richir: el presente (Gegenwart) "jamás adviene como tal si no es -mediante la transposición arquitectónica, y según el 'modelo' de la Stiftung de la apercepción perceptiva- en el acto de imaginar una imagen del propio objeto, imagen, por ende, ficticia e imaginaria"156. Advirtamos, al cabo, que esta temporalización en presencia sin presente no es del orden de una intencionalidad de acto, sino que,

de haber acto, éste se encuentra, a este nivel, originariamente disperso, es decir, parece ante la reflexión fenomenológica, declinado en plural originario, como siendo otros tantos 'momentos' abstractos de un 'obrar' o de un 'hacer' más global, y que corresponde al de la temporalización en presencia, 'hacer' en que la conciencia jamás coincide consigo misma en el Jetzt, en el ahora temporal del acto. ${ }^{57}$

¿Qué decir ahora, más específicamente, de la institución de la temporalidad de la propia apercepción de phantasia? Tal y como Husserl lo pone de manifiesto en el texto $n^{\circ} 1$ de Husserliana XXIII, las apercepciones de phantasia tienen, en cuanto a su temporalidad, un carácter discontinuo, intermitente y "proteiforme". ¿Cómo cabe interpretar, fenomenológicamente, dicho estado de cosas? La respuesta reside, precisamente, en la institución de la temporalidad específica de las apercepciones de phantasia (tal y como, paralelamente, el estatuto de las apercepciones perceptivas se explicaba mediante la institución de la temporalidad que les era específica). Dicho con mayor precisión, la apercepción de phantasia no instituye un presente aperceptivo (constitutivo de la permanencia y de la estabilidad de la forma de la "relación temporal bien ordenada"), sino un entreveramiento [enchevêtrement] de retenciones y de protenciones "sin cabeza" en un presente, o también de retenciones y protenciones "en la presen-

presencias y del presente de cada fase ha de ser completado en el sentido de que cada una de esas fases se caracteriza como fluyente". Ibidem, p. 100 (el subrayado es nuestro).

\footnotetext{
${ }^{55}$ PENF, p. 91.

${ }^{56}$ Ibidem, p. 92.

${ }^{57}$ Idem.
} 
$c^{\prime \prime a}{ }^{158}$. La estructura de la institución propia de la apercepción de phantasia reside, efectivamente, en el hecho de que no instituye presente alguno sino que, por el contrario, se caracteriza por la ruptura respecto del tiempo continuo del presente, es decir, que se caracteriza por sus surgimientos bruscos e inopinados, por estar siempre ligada como poco al esbozo, más o menos rápidamente abortado, de un sentido haciéndose, y por el hecho de que transpone arquitectónicamente lo inmemorial/inmaduro -cuyo rastro o testimonio portan las apariciones de phantasia (de las Wesen salvajes de lenguaje) - en "apercibido nunca visto" y en "apercibido por siempre invisible" en lo real ${ }^{59}$.

¿Cuál es pues, ahora, el estatuto de este entreveramiento retencionalprotencional desprovisto de cualquier presente asignable? ${ }^{60}$ M. Richir ve en todo ello "testimonios fenomenológicos" del campo fenomenológico más "arcaico" o más "primitivo" (desde un punto de vista arquitectónico) ${ }^{61}$, a saber, testimomios de esquirlas [éclats] dispersadas de temporalizaciones en presencia que jamás tuvieron lugar (y que resultan, así, oriundas de un "pasado inmemorial" y de un "futuro inmaduro"). Con todo, lo que hay que comprender -y llegamos por fin a la cuestión de la relación entre las entre- apercepciones de lenguaje y las apercepciones de phantasia-, es el vínculo, muy complejo, entre estas apercepciones de phantasia y los "Wesen salvajes" de lenguaje entendidos como "jirones [lambeaux]" fluctuantes e inestables de sentido haciéndose (por lo tanto, y para decirlo de una forma menos técnica, el vínculo entre la temporalización y la enunciación en lenguaje - "antes de" su institución en apercepción de lengua-). Efectivamente, las apercepciones de phantasia son "condensaciones simbólicas" de apercepciones de lengua (apercibiendo phantasia) -M. Richir llegará a decir que "la Stiftung de las apercepciones de phanta-

${ }^{58} \mathrm{Cfr}$. , por ejemplo, PENF, p. 251. M. Richir se explica una vez más sobre el particular en la página 258 (cita que resume el análisis nuestro que seguirá): "Estas apercepciones [de phantasia, que M. Richir denomina asimismo 'apercepciones en los límites (aperceptions aux limites)'] son sin presente, lo que quiere decir, para nosotros, que irreductiblemente son, y de modo originario, complejos de retenciones y de protenciones de lenguaje y de lengua a la vez 'sin cabeza' situada en presente alguno, no siéndolo tales retenciones y protenciones- sino de una presencia que se esboza, pero que suele eclipsarse por el juego, ya siempre transversalmente operativo en ella, de los ritmos de las síntesis pasivas".

${ }^{59}$ PENF, p. 259.

60 Precisemos que la noción de "presente" en la expresión "sin presente asignable" no tiene, para M. Richir, el mismo sentido que la "presencia" ("intrínseca") que caracteriza a la apercepción de phantasia. En el primer caso se trata del presente fijo y estable instituido por la apercepción perceptiva, mientras que en el segundo se trata de la "fluidez" de la presencia propia del tiempo originario (accesible, entre otras cosas, desde la apercepción de phantasia).

${ }^{61}$ Marc Richir denomina también a este registro el "propiamente fenomenológico", o el "campo fenomenológico stricto sensu". 
sia es estrictamente coextensiva con la Stiftung de la lengua"62 -, cuyas "condensaciones" resultan, a su vez, de la transposición arquitectónica del lenguaje en lengua ${ }^{63}$. La relación de mediación entre la "masa" (fuera de toda caracterización temporal y espacial) de los Wesen de lenguaje y la temporalización en presencia de las apercepciones de phantasia es entendida por M. Richir como una "codificación" que "estabiliza" los Wesen de lenguaje (y que es coextensiva con la transposición arquitectónica que transmuta los Wesen de lenguaje en apercepciones de lengua). Sin embargo, estos Wesen de lenguaje tampoco dejan de ser "reminiscencias transcendentales y premoniciones transcendentales del pasado por siempre inmemorial y del futuro por siempre jamás inmaduro de los esquematismos de lenguaje ${ }^{\prime 64}$. Y el vínculo (temporal) que existe entre los Wesen de lenguaje y las apercepciones de phantasia consiste no ya en su intemporalidad (lo cual podría sugerir su no-inscripción en el decurso temporal de la apercepción perceptiva) sino en lo que conforma su "potencialidad durmiente" (susceptibles, los primeros, de actualizarse en significaciones; los segundos, de surgir espontáneamente según el modo del Einfall) ${ }^{65}$, M. Richir escribe:

\begin{abstract}
Las síntesis pasivas [que suscitan las apercepciones de phantasia] no despiertan necesariamente tal o cual ritmo de temporalización en presencia depositado en el macizo del pasado; son, caso de no despertar nada, puros ritmos de temporalización en cierto modo por sí mismos y, de ese modo -al igual que un hablar que se inventa sobre la marcha- vuelven a poner en juego, en la temporalización en presencia de las apariciones de phantasia, toda la "masa" de los Wesen de lenguaje, y ello para ya instituir las apercepciones de phantasia en trance de temporalización en presencia. Significa esto [...] que esta "mobilización" de las apercepciones de lengua por las síntesis pasivas, su salida fuera de su estado de "potencialidades durmientes", no puede tener lugar [...] sino al albur de un sentido, todavía oscuro, partido en busca de sí mismo, y esbozando su temporalización en presencia. ${ }^{66}$
\end{abstract}

Disponemos en adelante de los elementos necesarios que nos permitirán comprender la institución de la intencionalidad en sentido husserliano. Al igual

62 PENF, p. 257.

${ }^{63}$ Recordemos que las apercepciones de phantasia no son idénticas a los Wesen de lenguaje (sino que son las concreciones fenomenológicas de los "Wesen salvajes") y que no hay congruencia entre las particiones [découpages] de las apercepciones de phantasia y las de las apercepciones de lengua (Cfr. el capítulo $\mathrm{V}$ de la $\mathrm{II}^{\mathrm{a}}$ sección de Phénoménologie en esquisses, en particular el § 4).

${ }^{64}$ PENF, p. 253.

${ }^{65}$ Cfr. a este propósito, PENF, pp. 256ss. Por otro lado, M. Richir pone de manifiesto otra correspondencia entre las apercepciones de lengua y las apercepciones de phantasia: de igual modo que la temporalización de las apercepciones de phantasia es intrínseca a su Stiftung, las apercepciones de lengua condensadas en "signos" remiten las unas a las otras en el interior de la enunciación lingüística; dicho de otro modo, así como la primera no requiere elemento alguno de procedencia externa (contrariamente a la apercepción perceptiva, que sí exige una "impresión originaria"), tampoco remite la última, eo ipso, a "referente" exterior alguno.

${ }^{66}$ Ibidem, p. 255. 
que Desanti, que ve en la impresión originaria (Urimpression) la piedra de toque de una comprensión coherente de la estructura fundante de la intencionalidad, también M. Richir considera que la institución de la intencionalidad no puede pasarse de un modelo explicativo que legitime esta impresión originaria. Al objeto de apuntalar nuestra reconstrucción de esta argumentación, necesitamos citar este largo pasaje que encierra todos los momentos necesarios a la comprensión de este modelo propuesto por M. Richir:

La transposición arquitectónica de las entre-apercepciones en entreapercepción formal y vacía del acuerdo entre ambos flujos (fuga en retenciones y resurgimiento a medida del ahora), y que conforma el presente vivo, transposición en la que, por lo tanto, el presente vivo se instituye, es consectaria -y es lo que se trata de comprender- del hecho de que el presente vivo sea ipso facto receptor de un contenido, pero de un contenido que le es exterior, que le llega, en la Urimpression, del afuera (y ello sin la suposición absurda de la "auto-afección pura" que es una ilusión transcendental fenomenológica); contenido que, por su parte, es apercibido de forma estable. Es pues como si la intención del sentido haciéndose se transpusiera arquitectónicamente, en virtud de la Stiftung de la apercepción perceptiva, en intencionalidad de la apercepción perceptiva, es decir, en intención del sentido intencional en su acepción husserliana, intención que, por "plenificada" que esté de intuiciones, jamás lo estará del todo, y cuya estructura (habitación recíproca de las intenciones vacías del pasado y de las intenciones vacías del futuro) sería la huella arquitectónica transpuesta de la habitación recíproca de las retenciones y de las protenciones propias del sentido haciéndose en su temporalización en presencia sin presente asignable ${ }^{67}$.

Como vemos, para dar cuenta de la intencionalidad en sentido husserliano, M. Richir recurre a una "transposición arquitectónica" que permite comprender la relación entre el "sentido haciéndose" y la intencionalidad de la apercepción perceptiva (al igual que el reajuste posible entre el flujo de "presentes vivos" y el de sus retenciones).

Para recapitular, proponemos el siguiente cuadro que permite visualizar la oposición entre los fenómenos que proceden de la temporalidad del sentido haciéndose y los que proceden de la de la apercepción perceptiva: hay transpo-

${ }^{67}$ Ibidem, p. 196. 
sición arquitectónica de las entre-apercepciones del sentido haciéndose en entre-apercepciones formales y vacías del acuerdo de ambos flujos:

\begin{tabular}{|c|c|}
\hline $\begin{array}{l}\text { Entre-apercepciones de lenguaje (tem- } \\
\text { poralidad del sentido haciéndose) }\end{array}$ & $\begin{array}{l}\text { Entre-apercepciones formales y vacías del acuer- } \\
\text { do de ambos flujos (temporalidad de la apercep- } \\
\text { ción perceptiva) }\end{array}$ \\
\hline temporalidad "concreta" & $\begin{array}{l}\text { temporalidad formal } \\
\text { no permanencia del objeto }\end{array}$ \\
base de las apercepciones de len- & $\begin{array}{l}\text { tuido por la forma del decurso temporal): su con- } \\
\text { tenido procede del exterior } \\
\text { gua no hay lenguaje (no hay elaboración } \\
\text { temporalizante del sentido haciéndose) }\end{array}$ \\
\hline
\end{tabular}

¿Cómo se opera entonces el paso de la entre-apercepción de lenguaje a la del acuerdo entre ambos flujos?

Ya hemos respondido, de hecho, a esta pregunta, ya que este paso corresponde precisamente al proceso de formalización que privará a la apercepción perceptiva de su contenido: "[...] La entre-apercepción del instante, inasible en su parpadeo, se formaliza como entre-apercepción del acuerdo que se rehace a medida en el flujo del decurso del presente, corto-circuitando a medida el desacuerdo sin cesar inminente como el estar-en-falso respecto de sí del sentido buscándose" ${ }^{\prime 68}$. Así, no queda sino el "tiempo 'puro' de lo Mismo, es decir, del presente vivo en flujo, ya muy alejado de la temporalidad concreta del sentido haciéndose, este Mismo que perdura a través de su propio flujo (acuerdo en flujo de ambos flujos) estando ya mucho más cerca de la intencionalidad"69. Asimismo, en virtud de este carácter formal (que todavía y de nuevo participa en la formación de sentido (Sinnbildung)) de la estructura de la apercepción perceptiva (resurgimiento y fuga de ambos flujos), ésta comporta en sí misma la huella (arquitectónicamente transpuesta) de las retenciones y de las protenciones entre-aperceptivas que obran en la formación del sentido haciéndose.

Sólo una vez sumergidos en las profundidades de la estructura de la intencionalidad se está en medida de comprender la estructura misma de los propios fenómenos. Cumple señalar que en Richir la fenomenalización no se confunde

${ }^{68}$ Ibidem, p. 195.

${ }^{69}$ Idem. 
en ningún caso con el aparecer (Erscheinen), ni tampoco con el juego de la aparición (Erscheinung) en el apareciente (Erscheinende); advertimos ahora lo que le permite a M. Richir sostener que esta fenomenalización no es otra cosa que el parpadeo (término en el cual resuena algo de la Schwingung heideggeriana) fenomenológico entre la aparición y la desaparición de fenómenos que parecen, de ese modo, como nada sino fenómenos (que no son fenómenos más que de sí mismos) ${ }^{70}$. Una épochè radical, precisamente la épochè "hiperbólica" (marrada por Husserl e incluso por el Fink de la VIa Meditación cartesiana) permite acceder, a la base del ego transcendental, a lo que conforma la unidad del doble-movimiento (del esquematismo transcendental) en el cual todo fenómeno, como nada sino fenómeno, parpadea indefinidamente e infinitamente entre su desaparición y su aparición: este doble-movimiento o este esquema mismo parpadea, en eco al parpadeo del fenómeno, entre su surgimiento como unidad, donde el ego transcendental se aparece, y su desvanecimiento como dispersión, donde el ego transcendental desaparece $[\ldots]^{71}$.

Lo de veras notable, en definitiva, es que a este campo último y "arcaico" de la fenomenología todavía pertenece un modo de "temporalidad" específico: el de lo instantáneo platónico (exaiphnès) "como virazón [revirement] incontrolable entre el movimiento y el reposo, el impulso [poussée] unificante y la dispersión"72. La épochè hiperbólica, en efecto, ya no sólo conlleva el suspenso instantáneo del decurso del tiempo objetivo (que se vuelve a poner de inmediato en flujo) ${ }^{73}$, ni tampoco el del tiempo "apareciente" (es decir, el del decurso temporal del presente vivo), sino antes bien el suspenso del "tiempo entero, y así sean cuales sean las estructuras de temporalización correlativas de un registro de pluralidad instituido (por Stiftung), y ello, cada vez, en lo instantáneo (exaiphnès) platónico $[\ldots]^{\prime \prime 74}$.

Comprobamos entonces cómo la tentativa de dar cuenta de la institución de la temporalidad inmanente -en términos richirianos: de la temporalidad de "la apercepción perceptiva"- nos franquea el acceso a una temporalidad absolutamente específica y que, precisamente, ya no procede de la mentada temporalidad inmanente misma, sino de una temporalización (propia de las apercepcio-

\footnotetext{
${ }^{70}$ Para todo esto, PENF, p. 23.

${ }^{71}$ Idem.

72 PENF, p. 24. Cfr. también p. 28 y p. 33.

${ }^{73}$ Ibidem, pp. 514ss.

${ }^{74}$ Ibidem, p. 28.
} 
nes de phantasia) ejecutiva en su relación originaria y última con la enunciación en lenguaje. En M. Richir, este análisis no es un fin en sí, sino que se inscribe en un ámbito de investigación más amplio y que incluye, notablemente, la institución de la idealidad (y también las de la "corporeidad propia" (Leiblichkeit) y de la intersubjetividad). Mas para poder dar razón de todo ello, antes hemos de estudiar la relación entre temporalidad y afectividad.

Traducido del francés por Pablo Posada Varela 